\section{PREVALENCE AND FACTORS ASSOCIATED WITH PHYSICAL ACTIVITY IN SOCIALLY VULNERABLE CHILDREN}

\author{
PREVALÊNCIA E FATORES ASSOCIADOSÀ ATIVIDADE FÍSICA DAS CRIANÇAS EM VULNERABILIDADE SOCIAL
}

PREVALENCIA Y FACTORES ASOCIADOS A LA ACTIVIDAD FISICA DE LOS NIÑOSEN VULNERABILIDADSOCIAL

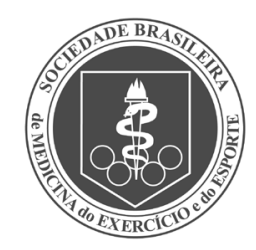

Original Article

Artigo OrIGINAL Artículo Original

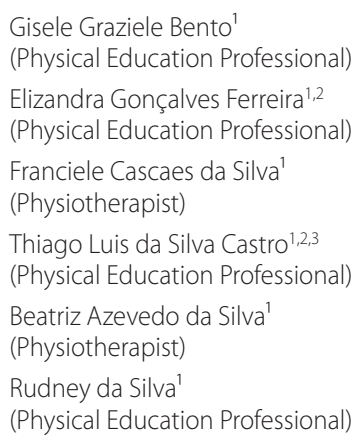

\section{Correspondence:}

Gisele Graziele Bento

Rua Pascoal Simone, 358,

Coqueiros, Florianópolis, SC, Brazil. 88080-350

giselegbento@gmail.com

\begin{abstract}
Introduction: Although the benefits of physical activity are widely recognized, insufficient levels have been identified among young people, especially those experiencing social vulnerability. Therefore, there is an ongoing search for health incentive strategies, especially in the early stages of life, aimed at reversing this situation. Objective: Investigate the prevalence of physical activity, weight status and motivation to participate in sports among socially vulnerable children who are participating in social projects of the state of Santa Catarina focused on sports, and compare these results according to age and sex. Methods: This is a cross-sectional descriptive study, composed of 3066 children aged seven to 11 years. Results: The study was made up of "active" and "moderately active" children of "normal weight", considering the domains of motivation to participate in physical activities and sports: pleasure, physical fitness, technical competence, general affiliation, specific affiliation and "very important" status and emotions and competition, "important". Accordingly, girls tend to be more "moderately active," "overweight" and "obese" and to regard technical competence as an "important" motivational domain for physical activity when compared to boys. Participants aged 10 to 11 years were more "very active", were more "overweight" and "obese" and considered specific affiliation more "totally important" when compared to younger participants. Conclusion: The participants of social sports projects of this sample demonstrated a level of physical activity and weight status within recommended levels, and were motivated to participate in sports in most of the domains observed. Level of evidence III; Diagnostic studies - Investigating a diagnostic test.
\end{abstract}

Keywords: Motor activity; Nutritional status; Motivation; Social vulnerability.

\section{RESUMO}

Introdução: Apesar de os benefícios da prática de atividade física serem amplamente reconhecidos, níveis insuficientes são constatados entrejovens, especialmente, aqueles em vulnerabilidade social. Assim, buscam-se estratégias de incentivo à saúde, especialmente, nas fases iniciais da vida, visando a reversão desse quadro. Objetivo: Investigar a prevalência da atividade física, status de peso e motivação para a prática esportiva das crianças em situação de vulnerabilidade social, participantes de projetos sociais na área esportiva do estado de Santa Catarina e comparar tais resultados de acordo com a idade e sexo. Métodos: Trata-se de um estudo transversal descritivo, composto por 3066 crianças de sete a 11 anos. Resultados: Composto por crianças "ativas" e "moderadamente ativas", com "peso normal', considerando-se os domínios motivacionais para a prática de atividades físicas e esportivas: prazer, condição física, competência técnica, afiliação geral, afiliação específica e status "muito importante" e emoções e competição, "importante". Desta forma, as meninas tendem a ser mais "moderadamente ativas", a apresentar "sobrepeso" e "obesidade" e a considerar a competência técnica como domínio motivacional "importante" à prática de atividade física quando comparadas aos meninos. Os participantes com 10 a 11 anos eram mais "muito ativos", apresentaram mais "sobrepeso" e "obesidade" e consideraram a afiliação específica mais "totalmente importante" quando comparados aos mais novos. Conclusão: Os participantes dos projetos sociais esportivos dessa amostra demonstraram um nível de atividade física dentro das recomendações, assim como o status de peso, sendo motivados à prática esportiva na maior parte dos domínios observados. Nível de evidência III; Estudos diagnósticos - Investigação de um exame para diagnóstico.

Descritores: Atividade motora; Estado nutricional; Motivação; Vulnerabilidade social.

\section{RESUMEN}

Introducción: A pesar de que los beneficios de la práctica de actividad física son ampliamente reconocidos, se han identificado niveles insuficientes entre jóvenes, especialmente aquellos en vulnerabilidad social. Así, se buscan estrategias de incentivo a la salud, especialmente en las fases iniciales de la vida, buscando la reversión de ese cuadro. Objetivo: Investigar la prevalencia de la actividad física, status de peso y motivación para la práctica deportiva de los niños en situación de vulnerabilidad social, participantes en proyectos sociales en el área deportiva del estado de Santa Catarina y comparar tales resultados según la edad y el sexo. Método: Se trata de un estudio transversal descriptivo, compuesto por 3066 niños de siete a 11 años. Resultados: Compuesto por niños "activos" "y "moderadamente activos" con "peso normal", considerándose los dominios motivacionales para la práctica de actividades físicas y deportivas: placer, condición física, competencia técnica, afiliación general, afiliación específica y status "muy importante" y emociones y competición, "importante". De esta forma, las niñas tienden a ser más "moderadamente activas", y 
presentar "sobrepeso" y "obesidad" y a considerar la competencia técnica como dominio motivacional "importante" para la práctica de actividad física cuando comparadas a los niños. Los participantes con 10 a 11 años eran más "muy activos", presentaron más "sobrepeso"y "obesidad"y consideraron la afiliación específica más "totalmente importante" cuando comparados a los más jóvenes. Conclusión: Los participantes de los proyectos sociales deportivos de esa muestra demostraron un nivel de actividad física dentro de las recomendaciones, asi como el status de peso, siendo motivados a la práctica deportiva en la mayor parte de los dominios observados. Nivel de evidencia III; Estudios

\section{diagnósticos - Investigación de un examen para diagnóstico.}

Descriptores: Actividad motora; Estado nutricional; Motivación; Vulnerabilidad social.

\section{INTRODUCTION}

Lifestyle has been undergoing changes in response to technological innovation, especially in the industrialized societies. ${ }^{1,2}$ Thus, childhood and adolescence are considered critical risk phases for physical inactivity and, consequently, excess body weight, ${ }^{3}$ especially in individuals at social risk due to cultural, educational, economic, and health differences. ${ }^{4}$

Although the benefits of physical activity are widely recognized, studies have identified that many children and adolescents do not reach sufficient levels of physical activity. ${ }^{3}$

Physical inactivity is associated with several physiological and psychological factors. ${ }^{5}$ Physiological factors mainly refer to increased body weight, which is associated with an increased prevalence of chronic degenerative diseases, such as cardiovascular, metabolic, and oncologic diseases, which are considered a public health problem. ${ }^{1,5}$

Psychological factors are related to behavioral changes that reduce the likelihood of adopting healthy habits as studies have shown that inactive children are more likely to become inactive adults ${ }^{3}$ and suffer from changes in mood and anxiety states that can cause several psychological disorders, such as depression and low self-esteem. ${ }^{5}$

To understand the motivation for the practice of physical and sport activities as a psychological factor, one must also understand the reasons that lead children and adolescents to engage in physical activities, which enables the design of more efficient intervention strategies. ${ }^{6,7}$

Socioeconomic factors are associated with social vulnerability, which creates conditions for certain groups, causing individual and collective risks that hinder the adoption of healthy habits. ${ }^{4}$ Studies have shown that children from the wealthier social classes are more likely to be physically inactive and overweight ${ }^{2}$ and have greater difficulties in accessing safe and appropriate spaces for physical activity, although such practices are a fundamental right. ${ }^{8}$

Considering the relevance of recognizing the various conditions related to physical activity during childhood $\mathrm{d}^{4,9}$ and its consequences for health, this study investigates the prevalence of physical activity, weight status, and motivation to practice physical and sport activities in socially vulnerable children who participate in social projects in the area of sports in the state of Santa Catarina and compares these results according to age and sex.

\section{MATERIALS AND METHODS}

This cross-sectional and descriptive study was approved by the Ethics Committee on Research in Humans of the State University of Santa Catarina under No. 087/2010.

A total of 3,066 socially vulnerable children aged from 7-11 years, of both sexes, participated in social projects in the area of sports and physical activity.

To ensure sample representativeness, we used official data showing that Brazil has 35,623,594 children aged from 7 to 11 years and the state of Santa Catarina has 387,342 in this age group. ${ }^{10}$ Thus, the sample size was calculated using the SampleXS for Windows, with the calculation of sample set for finite populations performed in two stages. ${ }^{11}$ In the first stage, the prevalence of physical activity was $80 \%$ in children, based on the specificity of the population, margin of error was lower than $0.5 \%$, and design effect was 2.0 points, adding 30\% for sample loss, which totaled 2424 subjects.

Of the 3,151 children, 85 cases were excluded from the sample due to lack of data in the questionnaire, totaling 3,066 children, with a sample loss of $2.7 \%$.

In the second stage, the groups were divided according to the regions proposed by the State Secretariat of Planning, ${ }_{1}^{12}$ using proportional stratified sampling. ${ }^{13}$ We calculated the sample fraction to determine the proportional sample, as shown in Table 1.

The instruments used were: 1) the Physical Activity Questionnaire - Older Children (PAQ-C) by Crocker et al., ${ }^{14}$ adapted into the Brazilian context by Guedes and Guedes ${ }^{15}$ and 2) the Participation Motivation Questionnaire (PMQ) by Gill, Gross, and Huddleston ${ }^{16}$ adapted by Serpa and Frias. ${ }^{17}$ We developed an extra questionnaire asking for sociodemographic, anthropometric, and health information. Anthropometric information comprised body weight and height of individuals. While body weight was measured using a Welmy brand mechanical scale that checked the body weight of subjects barefoot and using light clothes every 20 weighings, body height was measured to the nearest millimeter using a stadiometer with subjects in the orthostatic position, considering the classification of the World Health Organization. ${ }^{18}$

The professors coordinating the social project who would conduct the research in June 2010 were trained by LABAMA/CEFID/UDESC researchers.

Data were first tested for normality using the Kolmogorov-Smirnov test. The prevalence of physical activity, weight status, and motivation to practice were measured using descriptive statistics with simple and relative frequencies and a confidence interval of $95 \%$. These variables were compared for age and sex using the chi-square test.

Table 1. Projection of the sample by proportional group.

\begin{tabular}{c|c|c|c}
\hline Regions & Population & Estimated Sample & Final Sample \\
\hline West & 77,468 & 483 & 880 \\
\hline Northeast & 12,395 & 79 & 195 \\
\hline Planalto Norte & 20,142 & 127 & 311 \\
\hline Midwest & 38,734 & 242 & 199 \\
\hline Itajaí Valley & 40,284 & 253 & 179 \\
\hline Planalto Serrano & 17,043 & 108 & 424 \\
\hline South & 55,777 & 349 & 467 \\
\hline Coast & 125,499 & 783 & 3,066 \\
\hline Total & 387,342 & 2,424 & \\
\hline
\end{tabular}




\section{RESULTS}

Sociodemographic, anthropometric, sedentary behavior, and level of physical activity characteristics of the sample are shown in Table 2, which shows a predominance of boys aged from 10 to 11 years, living in the western region of the state of Santa Catarina in socioeconomic class C1. Regarding the time spent in sedentary behaviors, most individuals watched $<4$ hours of television per day. Most individuals were characterized as being active and moderately active and as having a normal weight.

Most domains of practice of physical and sport activities (pleasure, physical condition, technical competence, general affiliation, specific affiliation and status) were considered as "very important" by most participants, while emotions and competition were considered only as "important" (Table 3).

The results reveal that the level of physical activity is significantly associated with male sex, with boys being "active" and "very active," and girls predominantly being "moderately active" (Table 4).

The results also reveal that age is significantly associated with physical activity, with more subjects aged 10 to 11 years being "very active" than subjects aged between 7 and 9 years. (Table 4)

Table 2. Sociodemographic, anthropometric, sedentary behavior, and physical activity level characteristics of children participating in the study.

\begin{tabular}{|c|c|c|}
\hline Variable & $\mathrm{N}$ & $\%$ \\
\hline \multicolumn{3}{|c|}{$\operatorname{Sex}(n=3,066)$} \\
\hline Female & 1,453 & 47.3 \\
\hline Male & 1,613 & 52.6 \\
\hline \multicolumn{3}{|c|}{ Age $(n=3,066)$} \\
\hline 7-9 years & 1,039 & 33.9 \\
\hline 10-11 years & 2,027 & 77.1 \\
\hline \multicolumn{3}{|c|}{ Regions of state $(n=3,066)$} \\
\hline West & 880 & 28.7 \\
\hline Northeast & 195 & 6.4 \\
\hline Planalto Norte & 311 & 10.1 \\
\hline Midwest & 199 & 6.5 \\
\hline Itajaí Valley & 179 & 5.8 \\
\hline Planalto Serrano & 424 & 13.8 \\
\hline South & 411 & 13.4 \\
\hline Coast & 467 & 15.2 \\
\hline \multicolumn{3}{|c|}{ Socioeconomic classification $(n=3,064)$} \\
\hline Class E & 14 & 0.5 \\
\hline Class D & 246 & 8.0 \\
\hline Class C2 & 676 & 22.0 \\
\hline Class C1 & 1,032 & 33.7 \\
\hline Class B2 & 792 & 25.8 \\
\hline Class B1 & 269 & 8.8 \\
\hline Class A2 & 35 & 1.1 \\
\hline \multicolumn{3}{|c|}{ Classification of television watching hours $(n=3,037)$} \\
\hline Less than 4 hours & 2,413 & 79.4 \\
\hline More than 4 hours & 624 & 20.6 \\
\hline \multicolumn{3}{|c|}{ Classification of physical activity level $(n=3,066)$} \\
\hline Inactive & 36 & 1.2 \\
\hline Moderately active & 1,351 & 44.1 \\
\hline Active & 1,461 & 47.7 \\
\hline Very active & 218 & 7.1 \\
\hline \multicolumn{3}{|c|}{ Classification of weight status $(n=2,906)$} \\
\hline Low weight & 157 & 5.1 \\
\hline Normal weight & 2,046 & 66.7 \\
\hline Overweight & 503 & 16.4 \\
\hline Obesity & 200 & 6.5 \\
\hline
\end{tabular}

Weight affected boys less than girls. Boys had a predominantly "normal weight" or "low weight" status, and girls were predominantly "overweight" or"obese" (Table 5).

Age was significantly associated with weight, with more children aged 7-9 years having a "low weight" or "normal weight" than those aged 10-11 years (Table 5).

Motivation to practice sports and physical activities was only significantly associated with the female sex and the "important" technical competence (Table 6).

Motivation to practice sports and physical activities was significantly associated with age. More children aged 7-9 years old considered the status "less important"for the practice of physical activity and sports and competition compared to "totally important" for children aged10-11 years old. Conversely, more children aged 10-11 years old considered specific affiliation on "totally important" compared to children aged 7-9 years old (Table 5).

Table 3. Classification and prevalence regarding the motivation to practice physical and sports activities among children participating in the study $(n=3066)$.

\begin{tabular}{c|c|c|c|c|c}
\hline \multirow{2}{*}{ Domains } & N.I. & L.I. & I. & V.I. & T.I. \\
\cline { 2 - 6 } & $\mathbf{n}(\%)$ & $\mathbf{n}(\%)$ & $\mathbf{n}(\%)$ & $\mathbf{n}(\%)$ & $\mathbf{n}(\%)$ \\
\hline Status & $8(0,3)$ & $158(5.2)$ & $1,151(37.5)$ & $1,306(42.6)$ & $443(14.4)$ \\
\hline Emotions & $12(0,4)$ & $221(7.2)$ & $1,321(43.1)$ & $1,163(37.9)$ & $349(11.4)$ \\
\hline Pleasure & $1(0,0)$ & $56(1.8)$ & $834(27.2)$ & $1,365(44.5)$ & $810(26.4)$ \\
\hline Competition & - & $111(3.6)$ & $2,076(67.7)$ & $812(26.5)$ & $67(2.2)$ \\
\hline Physical condition & $2(0,1)$ & $69(2.3)$ & $827(27)$ & $1,408(45.9)$ & $760(24.8)$ \\
\hline Technical competence & $4(0,1)$ & $103(3.4)$ & $1,094(35.7)$ & $1,339(43.7)$ & $526(17.2)$ \\
\hline General Affiliation & $4(0,1)$ & $62(2.0)$ & $928(30.3)$ & $1,421(46.3)$ & $651(21.2)$ \\
\hline Specific Affiliation & $1(0,0)$ & $70(2.3)$ & $848(27.7)$ & $1,384(45.1)$ & $763(24.9)$ \\
\hline
\end{tabular}

n, Absolute frequency; \%, Relative frequency; N.I., not important; L.I., little important; l., important; V.I., very important; T.l., totally important.

Table 4. Comparison between sex, age, and physical activity level of children participating in the study.

\begin{tabular}{c|c|c|c|c|c|c}
\hline \multicolumn{7}{c}{ Classification according to the physical activity level } \\
\hline $\begin{array}{c}\text { Sex/age } \\
\mathbf{n}(\%)\end{array}$ & $\begin{array}{c}\text { Inactive } \\
\mathbf{n}(\%)\end{array}$ & $\begin{array}{c}\text { Moderately } \\
\text { active } \\
\mathbf{n}(\%)\end{array}$ & $\begin{array}{c}\text { Active } \\
\mathbf{n}(\%)\end{array}$ & $\begin{array}{c}\text { Very active } \\
\mathbf{n}(\%)\end{array}$ & $\mathbf{X}^{2}$ & $\mathbf{p}$ \\
\hline $\begin{array}{c}\text { Male } \\
1,613(52.6)\end{array}$ & $15(0.9)$ & $644(39.9)$ & $817(50.7)^{*}$ & $137(8.5)^{*}$ & 30,543 & 0.00 \\
\hline $\begin{array}{c}\text { Female } \\
1,453(47.3)\end{array}$ & $21(1.4)$ & $707(48.7)^{*}$ & $644(44.3)$ & $81(5.6)$ & & \\
\hline $\begin{array}{c}7-9 \text { years } \\
1,039(52.6)\end{array}$ & $17(1.6)$ & $470(45.2)$ & $495(47.6)$ & $57(5.5)$ & 9,178 & 0.02 \\
\hline $\begin{array}{l}10-11 \text { years } \\
2,027(47.3)\end{array}$ & $19(0.9)$ & $881(43.5)$ & $966(47.7)$ & $161(7.9)^{*}$ & & \\
\hline
\end{tabular}

$n$, Absolute frequency; $\%$, Relative frequency; $X^{2}$, chi square value; $p$, Significance level.

Table 5. Comparison between sex, age, and weight status of the children participating in the sample.

\begin{tabular}{c|c|c|c|c|c|c}
\hline \multicolumn{7}{c}{ Classification according to the weight status } \\
\hline $\begin{array}{c}\text { Sex/age } \\
\mathbf{n}(\%)\end{array}$ & $\begin{array}{c}\text { Low } \\
\text { weight } \\
\mathbf{n}(\%)\end{array}$ & $\begin{array}{c}\text { Normal } \\
\text { weight } \\
\mathbf{n}(\%)\end{array}$ & $\begin{array}{c}\text { Overweight } \\
\mathbf{n}(\%)\end{array}$ & $\begin{array}{c}\text { Obesity } \\
\mathbf{n}(\%)\end{array}$ & $\mathbf{X}^{2}$ & $\mathbf{p}$ \\
\hline $\begin{array}{c}\text { Male } \\
1,613(52.6)\end{array}$ & $118(7.3)^{*}$ & $1,489(92.3)^{*}$ & $0(0.0)$ & $6(0.4)$ & $1,299,207$ & 0.00 \\
\hline $\begin{array}{c}\text { Female } \\
1,293(47.3)\end{array}$ & $39(2.7)$ & $557(38.3)$ & $503(34.6)^{*}$ & $194(13.4)^{*}$ & & \\
\hline $\begin{array}{c}7-9 \text { years } \\
1,039(35.7)\end{array}$ & $66(6.4)^{*}$ & $968(93.2)^{*}$ & $0(0.0)$ & $5(0.5)$ & 597,012 & 0.00 \\
\hline $\begin{array}{c}10-11 \text { years } \\
1,867(64.3)\end{array}$ & $91(4.5)$ & $1,078(53.2)$ & $503(24.8)^{*}$ & $195(9.6)^{*}$ & & \\
\hline
\end{tabular}

$n$, Absolute frequency; \%, Relative frequency; $X^{2}$, chi-square value; $p$, Significance level. Analyzed by Fisher's Exact test. 
Table 6. Comparison between sex, age, and motivation to practice sports and physical activity of the children participating in the sample.

\begin{tabular}{|c|c|c|c|c|c|c|c|}
\hline Sex/age n(\%) & $\begin{array}{c}\text { N.I. } \\
\text { n(\%) }\end{array}$ & $\begin{array}{c}\text { L.I. } \\
\text { n(\%) }\end{array}$ & $\begin{array}{c}\mathrm{I} . \\
\mathrm{n}(\%)\end{array}$ & $\begin{array}{l}\text { V.I. } \\
\text { n(\%) }\end{array}$ & $\begin{array}{c}\text { T.I. } \\
\text { n(\%) }\end{array}$ & $X^{2}$ & $p$ \\
\hline \multicolumn{8}{|c|}{ Status } \\
\hline $\begin{array}{c}\text { Male 1,613 } \\
(52.6)\end{array}$ & $\begin{array}{c}5 \\
(0.3) \\
\end{array}$ & $79(4.9)$ & $\begin{array}{c}594 \\
(36.8)\end{array}$ & $\begin{array}{c}713 \\
(44.2)\end{array}$ & $\begin{array}{c}222 \\
(13.8)\end{array}$ & 4,380 & 0.357 \\
\hline $\begin{array}{c}\text { Female } 1,453 \\
(47.3)\end{array}$ & $\begin{array}{c}3 \\
(0.2)\end{array}$ & $79(5.4)$ & $\begin{array}{c}557 \\
(38.3)\end{array}$ & $\begin{array}{c}593 \\
(40.8)\end{array}$ & $\begin{array}{c}221 \\
(15.2)\end{array}$ & & \\
\hline $7-9$ years $1,039(52.6)$ & $\begin{array}{c}2 \\
(0.2)\end{array}$ & $\begin{array}{c}69 \\
(6.6)^{*}\end{array}$ & $\begin{array}{c}402 \\
(38.7)\end{array}$ & $\begin{array}{c}420 \\
(40.4)\end{array}$ & $\begin{array}{c}146 \\
(14.1)\end{array}$ & 9,499 & 0.05 \\
\hline $10-11$ years 2,027 (47.3) & $\begin{array}{c}6 \\
(0.3)\end{array}$ & $89(4.4)$ & $\begin{array}{c}749 \\
(37.0)\end{array}$ & $\begin{array}{c}886 \\
(43.7)\end{array}$ & $\begin{array}{c}297 \\
(14.7)\end{array}$ & & \\
\hline \multicolumn{8}{|c|}{ Emotions } \\
\hline $\begin{array}{c}\text { Male 1,613 } \\
(52.6)\end{array}$ & $\begin{array}{c}9 \\
(0.6) \\
\end{array}$ & $113(7.0)$ & $\begin{array}{c}677 \\
(42.0)\end{array}$ & $\begin{array}{c}628 \\
(38.9)\end{array}$ & $\begin{array}{c}186 \\
(11.5)\end{array}$ & 4,553 & 0.336 \\
\hline $\begin{array}{c}\text { Female 1,453 } \\
(47.3)\end{array}$ & $\begin{array}{c}3 \\
(0.2) \\
\end{array}$ & $108(7.4)$ & $\begin{array}{c}644 \\
(44.3) \\
\end{array}$ & $\begin{array}{c}535 \\
(38.8) \\
\end{array}$ & $\begin{array}{c}163 \\
(11.2) \\
\end{array}$ & & \\
\hline $\begin{array}{c}7-9 \text { years } 1,039 \\
(52.6)\end{array}$ & $\begin{array}{c}4 \\
(0.4)\end{array}$ & $81(7.8)$ & $\begin{array}{c}435 \\
(41.9)\end{array}$ & $\begin{array}{c}391 \\
(37.6)\end{array}$ & $\begin{array}{c}128 \\
(12.3)\end{array}$ & 2,545 & 0.637 \\
\hline $\begin{array}{c}\text { 10-11 years 2,027 } \\
(47.3)\end{array}$ & $\begin{array}{c}8 \\
(0.4) \\
\end{array}$ & $140(6.9)$ & $\begin{array}{c}886 \\
(43.7) \\
\end{array}$ & $\begin{array}{c}772 \\
(38.1) \\
\end{array}$ & $\begin{array}{c}221 \\
(10.9) \\
\end{array}$ & & \\
\hline \multicolumn{8}{|c|}{ Pleasure } \\
\hline $\begin{array}{c}\text { Male 1,613 } \\
(52.6)\end{array}$ & $\begin{array}{c}1 \\
(0.1)\end{array}$ & $\begin{array}{c}27 \\
(1.7) \\
\end{array}$ & $\begin{array}{c}420 \\
(26.0)\end{array}$ & $\begin{array}{c}732 \\
(45.4)\end{array}$ & $\begin{array}{c}433 \\
(26.8)\end{array}$ & 3,827 & 0.430 \\
\hline $\begin{array}{c}\text { Female } 1,453 \\
(47.3)\end{array}$ & - & $\begin{array}{c}29 \\
(2.0)\end{array}$ & $\begin{array}{c}414 \\
(28.5)\end{array}$ & $\begin{array}{c}633 \\
(43.6)\end{array}$ & $\begin{array}{c}377 \\
(25.9)\end{array}$ & & \\
\hline $\begin{array}{c}7-9 \text { years } 1,039 \\
(52.6)\end{array}$ & - & $\begin{array}{c}21 \\
(2.0) \\
\end{array}$ & $\begin{array}{c}285 \\
(27.4)\end{array}$ & $\begin{array}{c}479 \\
(46.1)\end{array}$ & $\begin{array}{c}254 \\
(24.4)\end{array}$ & 4,066 & 0.397 \\
\hline $\begin{array}{c}10-11 \text { years } 2,027 \\
(47.3)\end{array}$ & $\begin{array}{c}1 \\
(0.0) \\
\end{array}$ & $\begin{array}{c}35 \\
(1.7) \\
\end{array}$ & $\begin{array}{c}549 \\
(27.1) \\
\end{array}$ & $\begin{array}{c}886 \\
(43.7) \\
\end{array}$ & $\begin{array}{c}556 \\
(27.4) \\
\end{array}$ & & \\
\hline \multicolumn{8}{|c|}{ Competition } \\
\hline $\begin{array}{c}\text { Male 1,613 } \\
(52.6)\end{array}$ & - & $\begin{array}{c}62 \\
(3.8) \\
\end{array}$ & $\begin{array}{l}1,091 \\
(67.6) \\
\end{array}$ & $\begin{array}{c}423 \\
(26.2) \\
\end{array}$ & $\begin{array}{c}37 \\
(2.3) \\
\end{array}$ & 0,742 & 0.863 \\
\hline $\begin{array}{c}\text { Female 1,453 } \\
(47.3)\end{array}$ & - & $\begin{array}{c}49 \\
(3.4)\end{array}$ & $\begin{array}{c}985 \\
(67.8)\end{array}$ & $\begin{array}{c}389 \\
(26.8)\end{array}$ & $\begin{array}{c}30 \\
(2.1)\end{array}$ & & \\
\hline $\begin{array}{c}7-9 \text { years } 1,039 \\
(52.6)\end{array}$ & - & $\begin{array}{c}35 \\
(3.4)\end{array}$ & $\begin{array}{c}690 \\
(66.4)\end{array}$ & $\begin{array}{c}282 \\
(27.1)\end{array}$ & $\begin{array}{c}32 \\
(3.1)^{*}\end{array}$ & 6,680 & 0.08 \\
\hline $\begin{array}{c}10-11 \text { years } 2,027 \\
(47.3)\end{array}$ & - & $\begin{array}{c}76 \\
(3.7)\end{array}$ & $\begin{array}{l}1,386 \\
(68.4)\end{array}$ & $\begin{array}{c}530 \\
(26.1)\end{array}$ & $\begin{array}{c}35 \\
(1.7)\end{array}$ & & \\
\hline \multicolumn{8}{|c|}{ Physical condition } \\
\hline $\begin{array}{c}\text { Male 1,613 } \\
(52.6)\end{array}$ & $\begin{array}{c}2 \\
(0.1)\end{array}$ & $\begin{array}{c}32 \\
(2.0)\end{array}$ & $\begin{array}{c}434 \\
(26.9)\end{array}$ & $\begin{array}{c}753 \\
(46.7)\end{array}$ & $\begin{array}{c}392 \\
(24.3)\end{array}$ & 3,634 & 0.458 \\
\hline $\begin{array}{c}\text { Female 1,453 } \\
(47.3)\end{array}$ & - & $\begin{array}{c}37 \\
(2.5) \\
\end{array}$ & $\begin{array}{c}393 \\
(27.0)\end{array}$ & $\begin{array}{c}655 \\
(45.1)\end{array}$ & $\begin{array}{c}368 \\
(25.3)\end{array}$ & & \\
\hline $\begin{array}{c}\text { 7-9 years } 1,039 \\
(52.6)\end{array}$ & - & $\begin{array}{c}25 \\
(2.4)\end{array}$ & $\begin{array}{c}289 \\
(27.8) \\
\end{array}$ & $\begin{array}{c}471 \\
(45.3) \\
\end{array}$ & $\begin{array}{c}254 \\
(24.4) \\
\end{array}$ & 1,801 & 0.772 \\
\hline $\begin{array}{c}10-11 \text { years } 2,027 \\
(47.3)\end{array}$ & $\begin{array}{c}2 \\
(0.1)\end{array}$ & $\begin{array}{c}44 \\
(2.2)\end{array}$ & $\begin{array}{c}538 \\
(26.5)\end{array}$ & $\begin{array}{c}937 \\
(46.2)\end{array}$ & $\begin{array}{c}506 \\
(25.0)\end{array}$ & & \\
\hline \multicolumn{8}{|c|}{ Technical competence } \\
\hline $\begin{array}{l}\text { Male } 1,613 \\
(52.6)\end{array}$ & $\begin{array}{c}3 \\
(0.2)\end{array}$ & \begin{tabular}{|c|}
46 \\
$(2.9)$
\end{tabular} & $\begin{array}{c}544 \\
(33.7)\end{array}$ & $\begin{array}{c}725 \\
(44.9)\end{array}$ & $\begin{array}{c}295 \\
(18.3)\end{array}$ & 10,876 & 0.02 \\
\hline $\begin{array}{c}\text { Female 1,453 } \\
(47.3)\end{array}$ & $\begin{array}{c}1 \\
(0.1) \\
\end{array}$ & $\begin{array}{c}57 \\
(3.9)\end{array}$ & $\begin{array}{c}550 \\
(37.9)^{*}\end{array}$ & $\begin{array}{c}614 \\
(42.3) \\
\end{array}$ & $\begin{array}{c}231 \\
(15.9) \\
\end{array}$ & & \\
\hline $\begin{array}{c}\text { 7-9 years } 1,039 \\
(52.6)\end{array}$ & $\begin{array}{c}1 \\
(0.1)\end{array}$ & $\begin{array}{c}38 \\
(3.7)\end{array}$ & $\begin{array}{c}393 \\
(37.8)\end{array}$ & $\begin{array}{c}447 \\
(43.0)\end{array}$ & $\begin{array}{c}160 \\
(15.4) \\
\end{array}$ & 5,558 & 0.235 \\
\hline $\begin{array}{c}\text { 10-11 years 2,027 } \\
(47.3)\end{array}$ & $\begin{array}{c}3 \\
(0.1) \\
\end{array}$ & \begin{tabular}{|c|}
65 \\
$(3.2)$ \\
\end{tabular} & $\begin{array}{c}701 \\
(34.6) \\
\end{array}$ & $\begin{array}{c}892 \\
(44.0) \\
\end{array}$ & $\begin{array}{c}366 \\
(18.1) \\
\end{array}$ & & \\
\hline \multicolumn{8}{|c|}{ General affiliation } \\
\hline $\begin{array}{c}\text { Male 1,613 } \\
(52.6)\end{array}$ & $\begin{array}{c}3 \\
(0.2)\end{array}$ & \begin{tabular}{|c|}
29 \\
$(1.8)$
\end{tabular} & $\begin{array}{c}468 \\
(29.0)\end{array}$ & $\begin{array}{c}763 \\
(47.3)\end{array}$ & $\begin{array}{c}350 \\
(21.7)\end{array}$ & 4,436 & 0.350 \\
\hline $\begin{array}{c}\text { Female } 1,453 \\
(47.3)\end{array}$ & $\begin{array}{c}1 \\
(0.1)\end{array}$ & $\begin{array}{c}33 \\
(2.3)\end{array}$ & $\begin{array}{c}460 \\
(31.7)\end{array}$ & $\begin{array}{c}658 \\
(45.3)\end{array}$ & $\begin{array}{c}301 \\
(20.7)\end{array}$ & & \\
\hline $\begin{array}{c}7-9 \text { years } 1,039 \\
(52.6)\end{array}$ & $\begin{array}{c}2 \\
(0.2)\end{array}$ & $\begin{array}{c}24 \\
(2.3)\end{array}$ & $\begin{array}{c}305 \\
(29.4)\end{array}$ & $\begin{array}{c}496 \\
(47.7)\end{array}$ & $\begin{array}{c}212 \\
(20.4)\end{array}$ & 2,704 & 0.609 \\
\hline $\begin{array}{c}\text { 10-11 years 2,027 } \\
(47.3)\end{array}$ & $\begin{array}{c}2 \\
(0.1) \\
\end{array}$ & $\begin{array}{c}38 \\
(1.9) \\
\end{array}$ & $\begin{array}{c}623 \\
(30.7) \\
\end{array}$ & $\begin{array}{c}925 \\
(45.6) \\
\end{array}$ & $\begin{array}{c}439 \\
(21.7)\end{array}$ & & \\
\hline \multicolumn{8}{|c|}{ Specific affiliation } \\
\hline $\begin{array}{c}\text { Male 1,613 } \\
(52.6)\end{array}$ & $\begin{array}{c}3 \\
(0.2) \\
\end{array}$ & $\begin{array}{c}29 \\
(1.8) \\
\end{array}$ & $\begin{array}{c}468 \\
(29.0) \\
\end{array}$ & $\begin{array}{c}763 \\
(47.3) \\
\end{array}$ & $\begin{array}{c}350 \\
(21.7) \\
\end{array}$ & 4,436 & 0.350 \\
\hline $\begin{array}{c}\text { Female 1,453 } \\
(47.3)\end{array}$ & $\begin{array}{c}1 \\
(0.1) \\
\end{array}$ & $\begin{array}{c}33 \\
(2.3) \\
\end{array}$ & $\begin{array}{c}460 \\
(31.7) \\
\end{array}$ & $\begin{array}{c}658 \\
(45.3) \\
\end{array}$ & $\begin{array}{c}301 \\
(20.7) \\
\end{array}$ & & \\
\hline $\begin{array}{c}\text { 7-9 years } 1,039 \\
(52.6)\end{array}$ & $\begin{array}{c}2 \\
(0.2)\end{array}$ & $\begin{array}{c}24 \\
(2.3)\end{array}$ & $\begin{array}{c}305 \\
(29.4)\end{array}$ & $\begin{array}{c}496 \\
(47.7)\end{array}$ & $\begin{array}{c}212 \\
(20.4)\end{array}$ & 2,704 & 0.609 \\
\hline $\begin{array}{c}10-11 \text { years } 2,027 \\
(47.3)\end{array}$ & $\begin{array}{c}2 \\
(0.1) \\
\end{array}$ & $\begin{array}{c}38 \\
(1.9)\end{array}$ & $\begin{array}{c}623 \\
(30.7) \\
\end{array}$ & $\begin{array}{c}925 \\
(45.6) \\
\end{array}$ & $\begin{array}{c}439 \\
(21.7) \\
\end{array}$ & & \\
\hline
\end{tabular}

$n$, Absolute frequency; \%, Relative frequency; X2, chi square value; $p$, Significance level, N.I., not important; L.I, little important; I., important; V.I., very important; T.I., totally important.

\section{DISCUSSION}

We consider that the objectives of this study were achieved. The main limitations of this study were the fact that it was conducted by a large number of people, which impact we tried to minimize training and qualifying them; having used indirect measures for the physical activity level, which although are widely accepted and used, may limit the results, especially when it comes to self-recall in children; and the absence of other measures besides the BMI for the definition of nutritional status.

Socioeconomic conditions, along with health conditions, place of residence, education of parents, occupation of idle time, adoption of harmful habits to health, among others, is a factor that directly influences the social conditions of individuals being one of the causes of social vulnerability. ${ }^{4}$

Aiming at a more global understanding of the physical activity level, television watching time was used to measure the time spent in sedentary behaviors. The results obtained were below those found nationally, between 4 and 5 hours per day, and slightly above those found internationally. ${ }^{9}$ This finding may be because the survey participants' idle time is completely filled with projects for 3 days a week, as opposed to other national surveys. This also explains the value above the international average, as in these studies, the sample population had full-time education throughout the week.

Given that the participants were engaged in social projects in the sports area, we believed that all participants would be characterized as "active" and "very active". However, 36 children were considered physically "inactive". It is believed that these subjects may have had an eventuality that prevented their participation in the project in the target week or may have had a disability.

Studies have shown that in Brazil, the prevalence of physical activity varies according to the region of the study, from $27.1 \%{ }^{19}$ to $92.8 \% .^{20}$ The prevalence of physical inactivity ranges from $22.6 \%{ }^{21}$ to $93.5 \%{ }^{22}$ In Santa Catarina, researches have shown that physical inactivity affects $26-30 \%$ of children and adolescents, ${ }^{2,23}$ a result well above this study (1.2\%).

The positive association between male sex and the level of physical activity in all active classifications corroborates the findings in the literature. ${ }^{25}$ However, some studies indicate indifference between the sex or even greater physical activity in girls. ${ }^{24}$ In the latter, this comparison is more difficult because this was an international study with very different realities (Canada and Mozambique).

The finding that older participants were more "very active" than younger subjects contradicts the literature, which shows that age is inversely proportional to the level of physical activity. ${ }^{1}$ Such results can be attributed to the little difference between age groups and the activities offered to each of the groups in the projects.

With regard to the weight status, no participants were classified as "very low weight" and "severe obesity" according to the WHO classification criteria. ${ }^{18}$ Such extremes are not representative of the Brazilian child population, not being used in recent studies, corroborating the findings of this study. ${ }^{8}$

In Brazil, studies have indicated that the prevalence of overweight and obesity varies between $3.1 \%$ and $38.9 \% .{ }^{25}$ In the state of Santa Catarina, the prevalence ranges from $15.4 \%$ to $17.9 \%$ for overweight and from $6.0 \%$ to $6.7 \%$ for obesity, ${ }_{1}^{26}$ corroborating the findings of this study. According to the $\mathrm{IBGE}_{1}{ }^{10}$ there has been a sharp increase in the prevalence of overweight and obesity in in Brazil in the last three decades, from approximately $10.9 \%$ and $8.6 \%$ in the 1970 s to $15 \%$ and $12 \%$ in the 1980s and reaching 34.8\% and 32\% in 2008 and 2009 among boys and girls, respectively.

The results of the comparison between sex and weight status contradicts the findings by Duncan et al. ${ }^{27}$, who observed a prevalence between $10.2 \%$ and $19.4 \%$ for boys and $7.2 \%$ and $16.1 \%$ for girls. In 
contrast, Giugliano and Melo ${ }^{28}$, who conducted studies with the same population as this study, concluded that girls are associated with overweight and obesity, with a prevalence of $22.2 \%$. Overweight has also been associated with social vulnerability and low socioeconomic levels. ${ }^{2}$ Silva et al. ${ }^{23}$, when conducting a study in the state of Santa Catarina, observed a prevalence of overweight of $12.7 \%$ in boys and found that girls with low family income had a significant tendency to be overweight.

The study by Coelho et al. ${ }^{29}$ did not report significant differences between the children aged 7-9 years (9.03\%) and 10-14 years old (10.44\%) regarding relative body fat. Similarly, Carvalho et al. ${ }^{25}$ did not report significant differences in the age groups up to 6 years (35.4\%) and from $6-10$ years (38.9\%), indicating that $81.1 \%$ of children aged less than 6 years old and $49.3 \%$ of children over 6 years old were physically inactive. However, the study by Duncan et al. ${ }^{27}$ found that the likelihood of overweight and obesity were 0.36 times lower in children aged from 11 to 14 years, when compared to children aged from 7 to 10 years. Similarly, Siqueira et al. ${ }^{4}$ found higher percentages of overweight and obesity among children aged from 6 to 9 years (48\%).

Regarding the motivation to practice physical and sports activities, the study by Salguero, Gonzalez-Boto, and Tuero 6 points out that younger children show more interest in competition, friendship, ability, and status, corroborating the results of this study. It is worth mentioning that friendships mainly influence the motivation of participants through competitive and collaboration behaviors and social relations, especially in the initiation to sports. ${ }^{30}$ Most of these factors are related to the acceptance of peers, including technical competence and physical fitness, and the link between status and physical activity should be considered even more complex as some social groups are accepted more easily than those with a low level of physical activity. Moreover, Jago et al. ${ }^{30}$ reported that pleasure is considered the most important factor for the participation in physical activity by children of this age group.

Therefore, intervention through projects, such as that used in this study, that promote social inclusion using physical activity and healthy practices are suggested because, as pointed out by Sirard, Pfeiffer, and Pate $^{7}$, children who participate in this type of program are more active physically. Thus, to understand the motivation for physical activity is to improve the effectiveness of this type of program, meeting the preferences and priorities of its participants. 6,7

\section{CONCLUSION}

In this study, we investigated the prevalence of physical activity and associated factors in socially vulnerable children. We found that participants in our study sample, who participated in social projects in the sports area, had physical activity levels within the recommendations and well above those usually reported in the literature, as well as their weight status, being motivated to practice sports mainly by pleasure, physical condition, technical competence, general affiliation, specific affiliation, and status. Boys were more active than girls, and more girls were overweight and obese than boys, besides considering technical competence to be a more "important" motivational domain. Older participants were more "very active,"'overweight," and "obese" and considered specific affiliation to be more "totally important."

\section{All authors declare no potential conflict of interest related to this article}

AUTHORS' CONTRIBUTIONS: Each author made significant individual contributions to this manuscript. GGB (0000-0002-0006-3867)*: writing of the article, creation of the entire research project, critical review of its intellectual content and acquisition of data; EGF (0000-0003-1760-9704)*: statistical analysis; FCS (0000-0002-4444-9403)*: writing and statistics of the article; TLSC (0000-0002-2709-2422)*: writing and review of the article, interpretation of data for the work; BAS (0000-0003-4053-857X)*: writing; RS (0000-0002-9386-0039)*: substantial contribution to the conception or design of the work, interpretation of data for the work, critical review of its intellectual content. All authors approved the final version of the manuscript. *ORCID (Open Researcher and Contributor ID).

\section{REFERENCES}

1. Khoo S, Al-Shamli AK. Leisure-Time Physical Activity and Physical Fitness of Male Adolescents in Oman. Asia Pac J Public Health. 2012;24(1):128-35.

2. Pelegrini A, Petroski EL. Inatividade física e sua associação com estado nutricional, insatisfação com a imagem corporal e comportamentos sedentários em adolescentes de escolas públicas. Rev Paul Pediatr. 2009;27(4):366-73

3. Almeida PB, Silva V, Cyrino ES. Perfil antropométrico de crianças e adolescentes atendidos por unidades educacionais na periferia do município de Londrina-PR. Rev Bras Cien e Mov. 2009;17(3):1-8.

4. Siqueira FV, Nahas MC, Facchini IA, Silveira DS, Piccini RX, Tomasi E, et al. Aconselhamento para a prática de atividade física como estratégia de educação à saúde. Cad. Saude Publica. 2009;25(1):203-13.

5. Rennie KL, Johnson L, Jebb SA. Behavioural determinants of obesity. Best Pract Res Clin Endocrinol Metab. 2005;19(3):343-58

6. Salguero A, Gonzalez-Boto R, Tuero C, Márquez S. Development of a Spanish version of the Participation Motivation Inventory for young competitive swimmers. Percept Mot Skills. 2003;96(2):637-46.

7. Sirard JR, Pfeiffer KA, Pate RR. Motivational factors associated with sports program participation in middle school students. J Adolesc Health. 2006;38(6):696-703

8. Stanley RM, Boshoff K, Dollman J.Voices in the playground: a qualitative exploration of the barriers and facilitators of lunchtime play. J Sci Med Sport. 2012;15(1):44-51.

9. da Silva RC, Malina RM. Nível de atividade física em adolescentes do Município de Niterói, Rio de Janeiro, Brasil. Cad. Saude Publica. 2000;16(4):1091-7.

10. Instituto Brasileiro de Geografia e Estatística. Censo 2010 - 2011. [acesso em 2011 out 20]. Disponível em: http://www.ibge.gov.br

11. Kumar R. Research Methodology: A step-by-step guide for beginners. London: SAGE Publications; 1996.

12. Santa Catarina. Regiōes geoeconômicas do Estado. Página do Governo do Estado de Santa Catarina. [acesso em 2009 nov 12]. Disponível em: http://www.sc.gov.br/conteudo/santacatarina/geografia/ paginas/regioes.htm

13. Barbetta PA. Estatística aplicada às ciências sociais. Florianópolis: Ed. UFSC; 2003.

14. Crocker PR, Bailey DA, Faulkner RA, Kowalski KC, Mcgrath R. Measuring general levels of physical activity: preliminary evidence for the Physical Activity Questionnaire for Older Children. Med Sci Sports Exerc 1997;29(10):1344-9.

15. Guedes DP, Guedes JE. Medida da atividade física em jovens brasileiros: reprodutibilidade e validade do PAQ-C e do PAQ-A. Rev Bras Med Esporte. 2015;21(6):425-32.
16. Gill DL, Gross JB, Huddleston S. Participation motivation in youth sports. Int. J. Sports Psychol. 1983;14:1-14. 17. Serpa S, Frias J. Estudo da relação professor/aluno em ginástica de representação e manutenção. Monografia [Graduação] - Faculdade de Motricidade Humana, Universidade Técnica de Lisboa; 1990.

18. Organização Mundial da Saúde. BMI-for-age GIRLS: 5 to 19 years (z-scores). Geneva:WHO; 2007.

19. Mazaro IA, Zanolli Mde L, Antonio MA, Morcillo AM, Zambon MP. Obesity and cardiovascular risk factors in school children from Sorocaba, SP. Rev Assoc Med Bras. 2011;57(6):674-80.

20. Costa SM, Horta PM, dos Santos LC. Food advertising and television exposure: influence on eating behavior and nutritional status of children and adolescents. Arch Latinoam Nutr. 2012;62(1):53-9.

21. Ribeiro RQ, Lotufo PA, Lamounier JA, Oliveira RG, Soares JF, Botter DA. Additional cardiovascular risk factors associated with excess weight in children and adolescents: the Belo Horizonte heart study. Arq Bras Cardiol. 2006;86(6):408-18.

22. Rivera IR, Silva MA, Silva RD, Oliveira BA, Carvalho AC. Physical inactivity, TV-watching hours and body composition in children and adolescents. Arq Bras Cardiol. 2010;95(2):159-65.

23. Silva HG, Chiara VL, Barros ME, Rêgo AL, Ferreira A, Pitasi BA, et al. Diagnosing the nutritional status of schoolchildren: a comparison between Brazilian and international criteria. J. Pediatr (Rio J). 2008;84(6):550-5.

24. Nhantumbo L, Maia J, Saranga S, Prista A. Atividade física em crianças e jovens residentes em uma comunidade rural moçambicana: efeitos da idade, sexo e estado nutricional. Rev Panam Salud Publica. 2008;23(3):171-8

25. de Carvalho Cremm E, Marrocos LF, de Abreu DS, de Oliveira MA, Scagliusi FB, Martins PA. Factors associated with overweight in children living in the neighbourhoods of an urban area of Brazil. Public Health Nutr. 2012;15(6):1056-64.

26. Soar C, Vasconcelos Fde A, Assis MA. A relação cintura quadril e o perímetro da cintura associados ao índice de massa corporal em estudo com escolares. Cad. Saude Publica. 2004;20(6):1609-16.

27. Duncan S, Duncan EK, Fernandes RA, Buonani C, Bastos KD, Segatto AF, et al. Modifiable risk factors for overweight and obesity in children and adolescents from São Paulo, Brazil. BMC Public Health. 2011;11:585.

28. Giugliano R, Melo AL. Diagnóstico de sobrepeso e obesidade em escolares: utilização de massa corporal segundo padrão internacional. J Pediatr (Rio J). 2004;80(2):129-34.

29. Coelho LG, Cândido AP, Machado-Coelho GL, Freitas SN. Association between nutritional status, food habits and physical activity level in schoolchildren. J Pediatr (Rio J). 2012;88(5):406-12.

30. Jago R, Brockman R, Fox KR, Cartwright K, Page AS, Thompson JL. Friendship groups and physical activity: qualitative findings on how physical activity is initiated and maintained among 10-11 year old children. Int J Behav Nutr Phys Act. 2009;6:4. 